\title{
Evaluation of a Vibration Text Fixture
}

\author{
Everaldo de Barros and Carlos d'Andrade Souto \\ Institute of Aeronautics and Space, Praça Eduardo Gomes, 50, 12228-904 São José dos Campos, SP, Brazil.
}

\begin{abstract}
(Received 9 December 2014; accepted 3 May 2016)
Mechanical vibration tests require the use of vibrators, power amplifiers, accelerometers, and closed loop control systems. A device is needed to connect physically the specimen to be tested to the vibrator table referred to as vibration test fixture. To verify and validate a fixture to be used, an evaluation of its dynamical behaviour is mandatory. The fixture natural frequencies must be higher than the maximum test frequency. In this work, some issues about electrodynamic vibrators and fixtures are revised and the behaviour of a real fixture is analysed numerically and experimentally. The fixture natural frequencies and its response to a harmonic acceleration applied on its bottom surface are evaluated by using the finite element method. A real test was performed by applying slow swept sine acceleration to the fixture mounted in an electrodynamic vibrator and measuring its response. The effect of the closed loop control system on the fixture response is evaluated. It is shown that the control system plays an important role in the definition of the fixture useful operating frequency range.
\end{abstract}

\section{INTRODUCTION}

Some products are exposed to severe noise and vibration loads during their life cycle. Thus, it is mandatory to perform tests that simulate the product with the environment loads when the product is still in the development phase to verify whether it can withstand the loads keeping its performance under acceptable levels. Mechanical vibration tests evaluate the product structural dynamics behaviour. In a mechanical vibration test, the device under test (DUT) is submitted to predetermined levels of vibration in order to simulate its life cycle operation environment. The essential components to such a test are: the vibrator, the power amplifier, accelerometers, a closed loop control system and a fixture, a device that connects the DUT, and the vibrator moving part. The function of the power amplifier is to supply the vibrator with electric power necessary to generate vibration motion. In simple terms, more velocity requires more voltage and more force or acceleration requires more current from the power amplifier. Accelerometers are transducers able to measure the vibration motion, and during vibration tests they are used to verify the vibratory levels reached by some DUT regions. The control system operates in a closed loop and its function is to check and assure that the vibration levels on the vibrator table are according to the specified values for the test. The feedback signal is provided by accelerometers located on the vibrator table or on the control points of the DUT. The control system governs the vibration table motion and, if necessary, corrects its output in order to follow the pre- determined vibration profile level. Finally, the fixture function is to attach the DUT to the vibrator table and transmit the vibratory motion to the DUT with minimum interference.

Some research has been carried out on the dynamic characteristics of test fixtures. For example, the dynamic characteristics of fixtures for space launch vehicles vibration test are discussed by Reddy and Reddy. ${ }^{1}$ Considerations for vibration fixture design are discussed by Avitable. ${ }^{2}$ Girard, et al., ${ }^{3}$ presented the design analysis and experimental results to confirm the dynamic properties of a test fixture designed to support large specimens in axial configuration. A test fixture is modelled and analysed by using finite element analysis by Sowjanya, et al. ${ }^{4}$ Some methods are proposed on structural dynamics modification for vibration test fixtures. ${ }^{5,6}$ Methods of structural dynamic response optimization of fixtures have been reported. ${ }^{7,8}$ A complete background on fixture design for vibration and shock testing can be found in the literature. 9,10 Ideally, a fixture couples the motion from the vibrator to the DUT with high fidelity, with zero distortion at all amplitudes and frequencies of interest. In practice, weight, size, high natural frequency, and cost considerations are the major factors that greatly affect the design and built of vibration test fixtures. Many times, fixtures were designed by using empirical methods, not taking into account its dynamic characteristics. ${ }^{7}$ The dynamic behaviour of complex structures such as vibration test fixtures is often far different from what has been calculated and usually, the ideal cannot be met and limitations of the fixtures must be known. ${ }^{10}$ This means that new fixture experimental evaluation is required before using it in test programs. This procedure will avoid situations where the test will fail at certain frequencies.

Following this recommendation, this work deals with a very relevant topic, being the correct use of fixtures in dynamic testing. In order to enhance the requirements of quality of vibration test programs conducted at various laboratories, this paper presents a methodology to verify the dynamic performance of already built fixtures. This work is focused on the dynamic behaviour analysis of an already built specific vibration test fixture. The dynamic performances are analysed numerically and experimentally. The most appropriate frequency range for vibration tests and regions on the fixture to fix the DUT are defined.

The experimental obtaining of the transmissibility on various points of the fixture allowed the definition of: a) of the fixture useful area to meet the requirements of the dynamic test- 


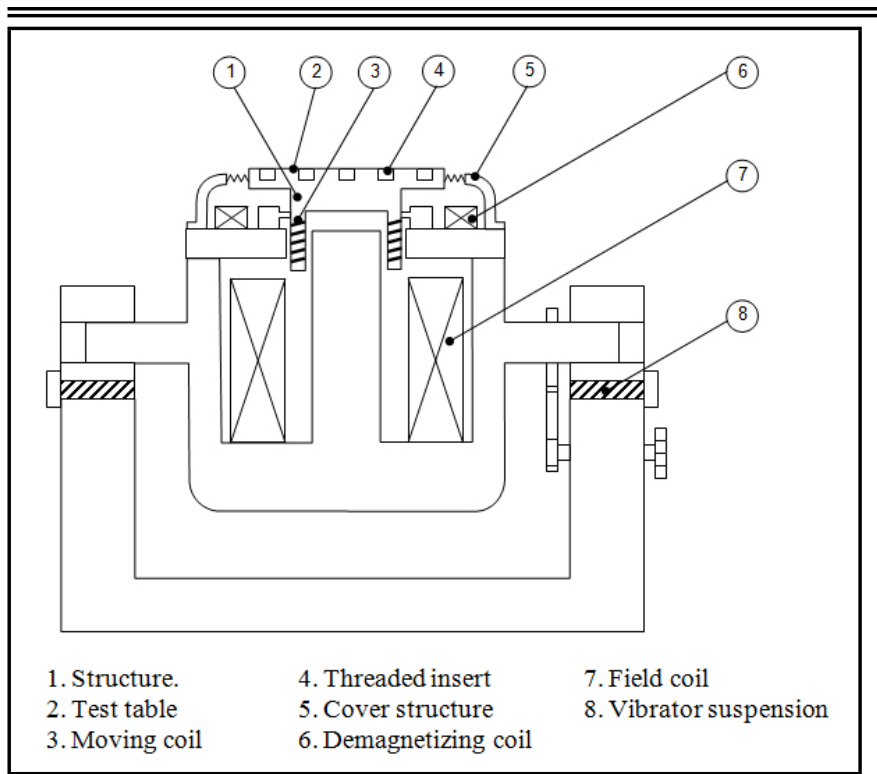

Figure 1. Typical arrangement of an electrodynamic vibrator.

ing; b) the permissible operating frequency range over which testing is to be conducted taken into account the shaker armature dynamic These conclusions lead to rich control in the test specification output and indicate that this methodology is essential for evaluating the vibration text fixtures.

\section{ELECTRODYNAMIC VIBRATORS}

An electrodynamic vibrator works similarly to a loudspeaker, where the axial movement of the coil, referred to as the armature, is produced by an electrical current, provided from a power amplifier. The vibrator force is generated by the interaction between the current flowing through the armature and the static magnetic field. This magnetic field is produced by a permanent magnet (for small forces) or by an electromagnet (for large forces). The electromagnet is referred to as the field coil.

When the current varies harmonically in time, the generated force varies in the same manner. Due to this, the force generated by an electrodynamic vibrator varies according to the input signal and then can be periodic, transient or random, or a combination of each. In this way, it is straightforward to obtain excitations of very different nature from electrodynamic vibrators in dynamic tests. A cross section of an electrodynamic vibrator with field coil is shown in Fig. 1. ${ }^{11}$

The typical frequency response of an electrodynamic vibrator, for a constant amplitude input voltage applied to the drive coil, can be seen in Fig. 2, where $f_{0}$ is the electrical resonance frequency damped by the armature resistance and $f_{1}$ is the axial mechanical resonance frequency of the armature. ${ }^{12}$ The upper limit frequency is determined by the axial mechanical resonance frequency of the vibrator and it is recommended to operate at frequencies lower than $f_{1}$. Commercially available electrodynamic vibrators cover the useful operating frequency range from $5 \mathrm{~Hz}$ to $2000 \mathrm{~Hz}$, from either a sinewave or random signal input.

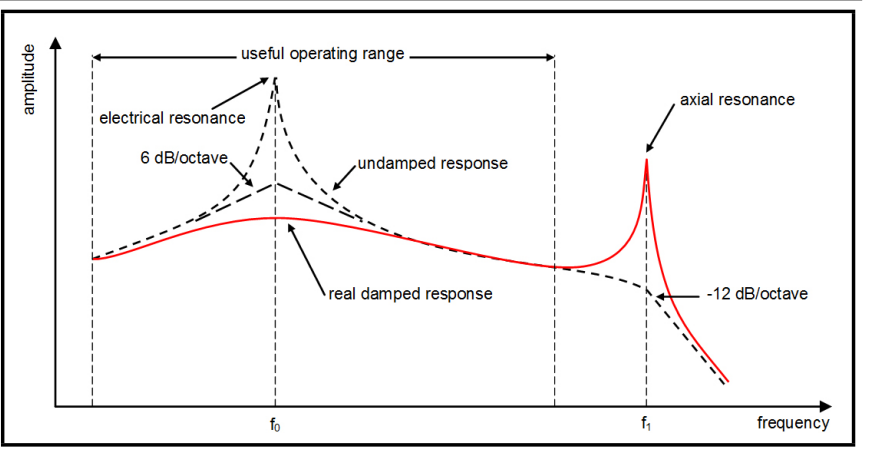

Figure 2. Typical frequency response of an electrodynamic vibrator for a constant amplitude input voltage.

\section{VIBRATION TEST FIXTURES}

When making the design of a fixture one should analyse its transmissibility and resonance frequencies. ${ }^{13}$ A vibration test fixture must have high stiffness and be able to transfer the vibratory energy generated by the vibrator to the DUT as unchanged as possible. In other words, the vibratory energy in the fixture's output should be the same in the fixture's input, where the vibrator is connected, for all the investigated frequency range. Basically, the fixture should be as light as possible, as stiff as possible, should have unitary transmissibility in the investigated frequency range and its resonance frequencies should be beyond the investigated frequency range.

In order to design a fixture, it is necessary to take into account the kind of test (or tests) to be performed (e.g. vibration or shock); the DUT characteristics (its dynamics and the way it is mechanically attached to the vibrator), and the test equipment characteristics (amplifier power; maximum values of displacement acceleration and velocity, force, and admissible mass over the armature).

In the design phase, the different fixture parameters can be related and a compromise among the resonance frequencies, mass, shape, fastening, and material damping need to be achieved. Since high values of resonance frequencies are desired, the fixture must be stiff enough. However, the maximum acceleration provided by the vibrator to the DUT depends on the total moving mass (fixture + DUT + armature). Once the DUT and armature masses are constant, the fixture mass must be as small as possible. Since there is a need for reducing the mass and amplifying the resonance frequencies, the use of alloys of aluminium and magnesium is recommended because they present low specific mass and high structural damping (compared to steel).

\section{CASE STUDY}

The analysed test fixture is shown in Fig. 3. It was manufactured in an aluminium alloy and has a diameter of $330 \mathrm{~mm}$, with a maximum thickness of $33.4 \mathrm{~mm}$. It has 13 bolt holes (M8 standard) and it weighs $5.956 \mathrm{~kg}$. This fixture was developed specifically to a LDS model 824 vibrator, whose characteristics are described in Table 1. 


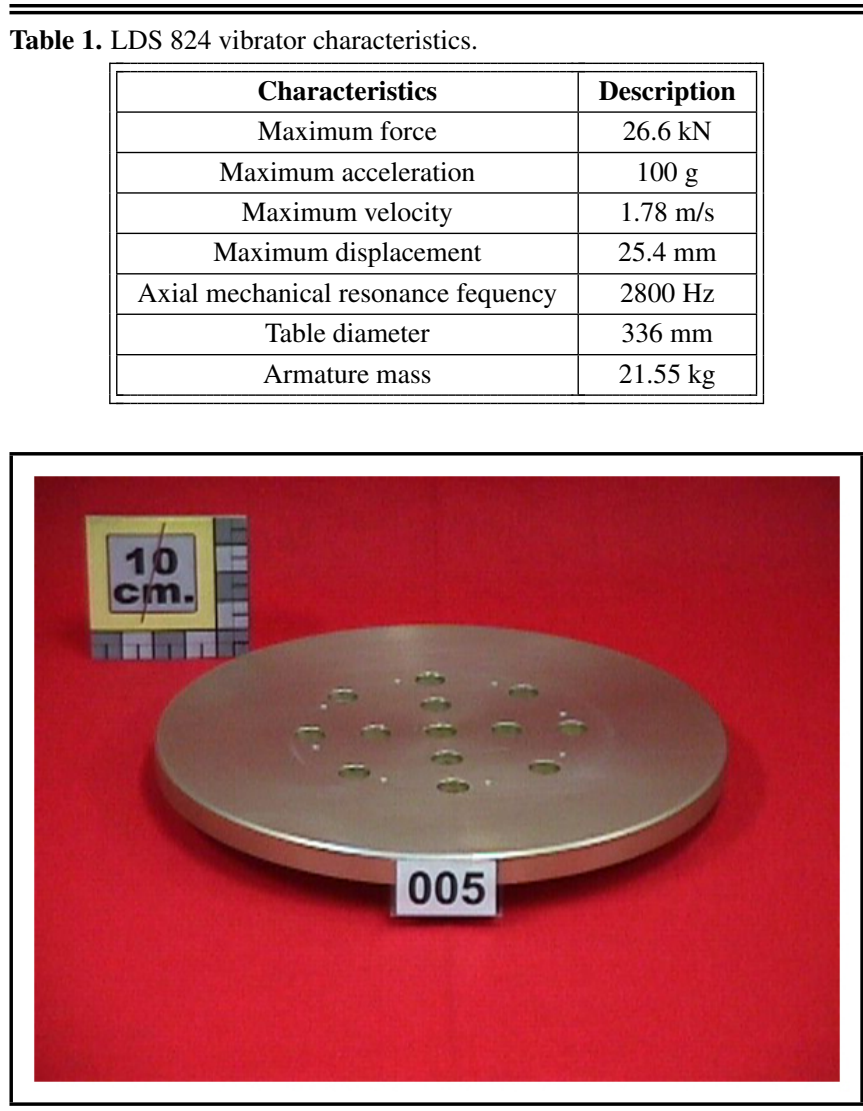

Figure 3. Fixture analyzed.

\subsection{Analysis by Using Finite Elements}

The fixture natural frequencies were calculated by using the finite element package ANSYS. The fixture is fixed to the shaker table by bolts. In the FEM model, zero displacement boundary condition was applied to the nodes placed on the bolt holes. The fixture volume was discretized using linear tetrahedral elements, ${ }^{14}$ in a mesh with 5420 nodes, 24748 elements (a total of 16,260 degrees of freedom). A view of the mesh is displayed in Fig. 4. The fixture natural frequencies calculated are listed in Table 2. As will be described in Section 4.2, the fixture was submitted to a vibration test with the application of a swept sine acceleration in a frequency range from $10 \mathrm{~Hz}$ to $2500 \mathrm{~Hz}$, with constant amplitude of $0.5 \mathrm{~g}\left(g=9.81 \mathrm{~m} / \mathrm{s}^{2}\right)$. This test was also simulated numerically and the frequency responses were calculated in some nodes of the finite element model, corresponding to the points where the responses were measured in the test $(1,2$, and 3, shown in Fig. 5).

The response frequencies in the three points are shown in Fig. 6. It was stated before that the fixture should present a

Table 2. LDS 824 vibrator characteristics.

\begin{tabular}{|c|c|}
\hline Mode No. & Frequency $(\mathbf{H z})$ \\
\hline 1 & 1818.31 \\
\hline 2 & 1818.31 \\
\hline 3 & 1916.64 \\
\hline 4 & 2265.28 \\
\hline 5 & 2270.99 \\
\hline 6 & 2823.47 \\
\hline 7 & 3916.44 \\
\hline 8 & 3916.45 \\
\hline
\end{tabular}

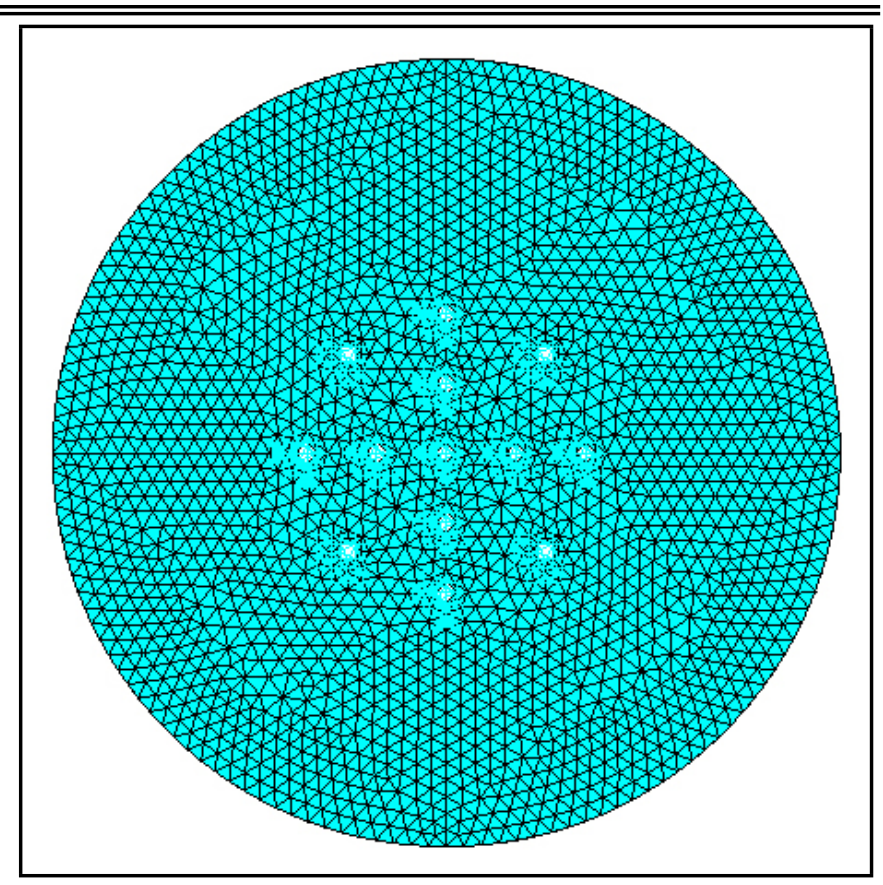

Figure 4. Mesh used in the analysis.

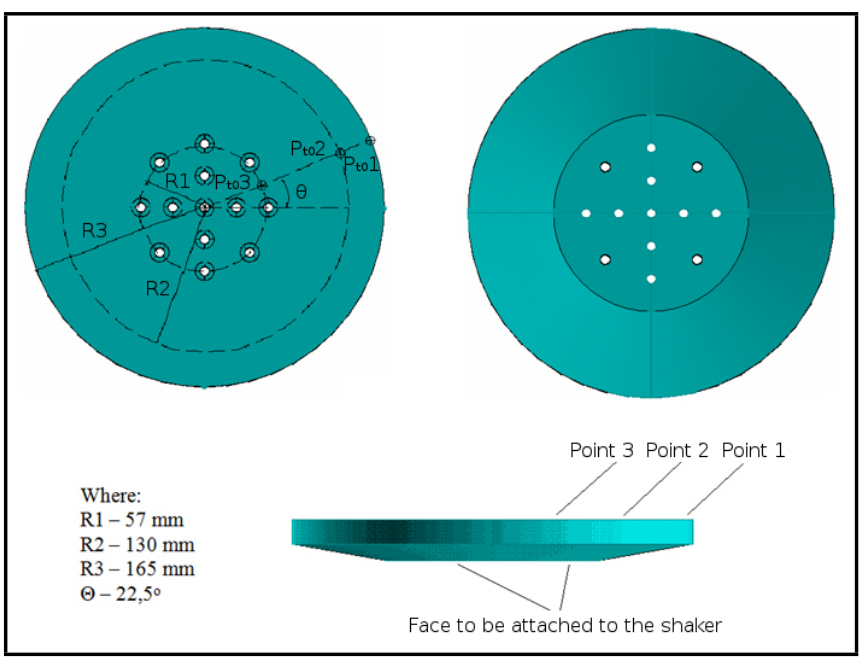

Figure 5. Control accelerometers positions on the fixture.

transmissibility of value one. The fixture's transmissibility of the i-th point can be written as:

$$
T_{i}(\omega)=\frac{\operatorname{Acel}_{i}(\omega)}{\operatorname{Acel}_{\text {ref }}(\omega)}
$$

where $T_{i}(\omega)$ is the transmissibility in point $\mathrm{i}, \operatorname{Acel}_{i}(\omega)$ is the acceleration response frequency calculated in point $i$, and Acel $_{\text {ref }}(\omega)$ is the acceleration applied to adapter's bottom. By using this equation and the response frequencies shown in Fig. 6, the following acceleration transmissibility as a function of frequency in points 1, 2, and 3 (shown also in Fig. 6) is obtained.

\subsection{Control System Performance}

For the evaluation, the fixture was mounted on the vibrator table and was submitted to a test with the application of a swept sine acceleration in a frequency range from $10 \mathrm{~Hz}$ to $2500 \mathrm{~Hz}$, with constant amplitude of $0.5 \mathrm{~g}\left(g=9.8 \mathrm{~m} / \mathrm{s}^{2}\right)$. The 


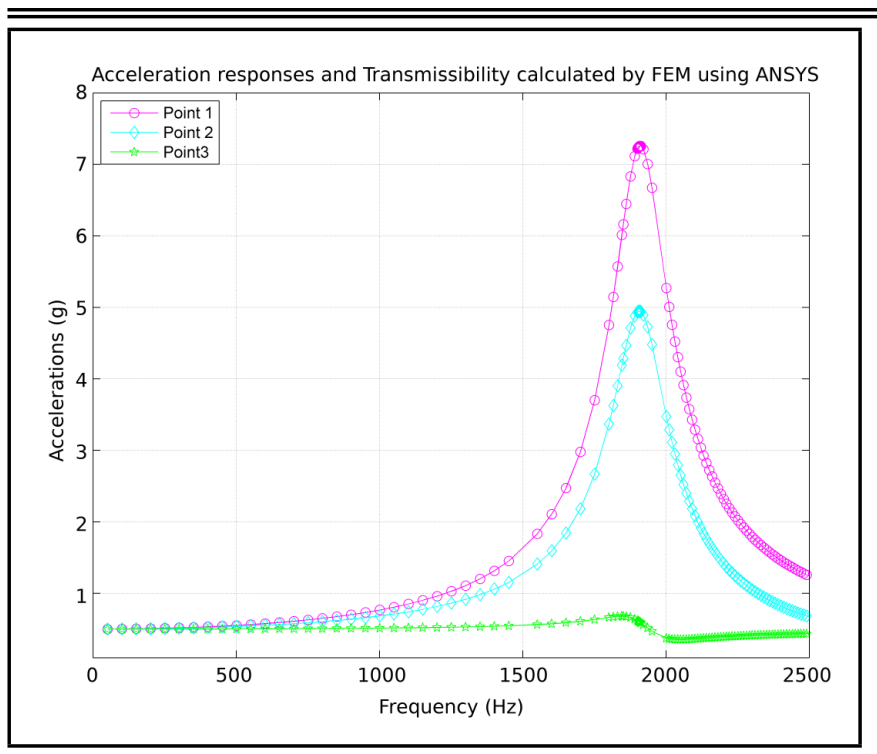

Figure 6. Frequency response-Transmissibility calculated by using ANSYS.

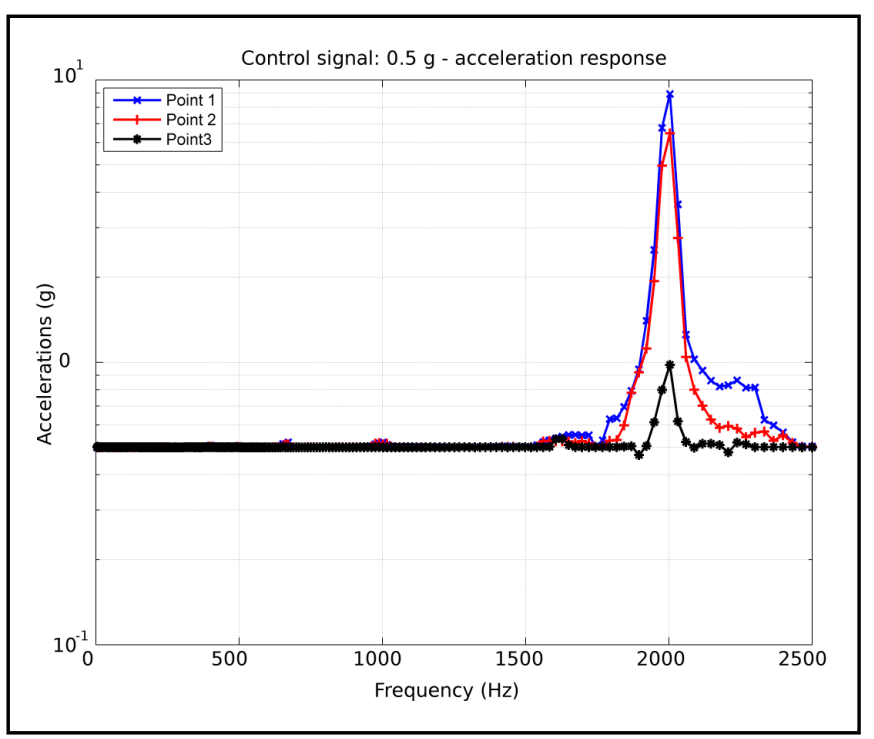

Figure 7. Frequency response of control accelerometer for the control points 1,2 , and 3 .

sweeping rate was 1 octave/minute. The control security level was specified in $\pm 3 \mathrm{~dB}$ (from $0.354 \mathrm{~g}$ to $0.706 \mathrm{~g}$ ). Three runs were performed placing the control accelerometer in the points 1,2 , and 3, respectively. The control accelerometer used was a Brel\&Kjaer model 4375 used with a Brel\&Kjaer model 2626 signal conditioner. The frequency response provided by control accelerometer and the frequency response of the control system output (drive signal) are shown in Fig. 7 and Fig. 8, respectively, for the control points 1,2 , and 3 . It can be realized by analysing Fig. 8 that the control system output signal reaches minimum value at $2000 \mathrm{~Hz}$ (close to the bolted fixture $3^{\text {rd }}$ mode frequency) for all three control accelerometer positions. This indicates that the control system was trying to reduce the vibration level measured by the control accelerometer but this action, however, was not able to avoid the vibration level to reach and overtake the security limit pre-defined for the test $(0.706 \mathrm{~g})$.

Actually, the vibration levels orthogonal to fixture's top face

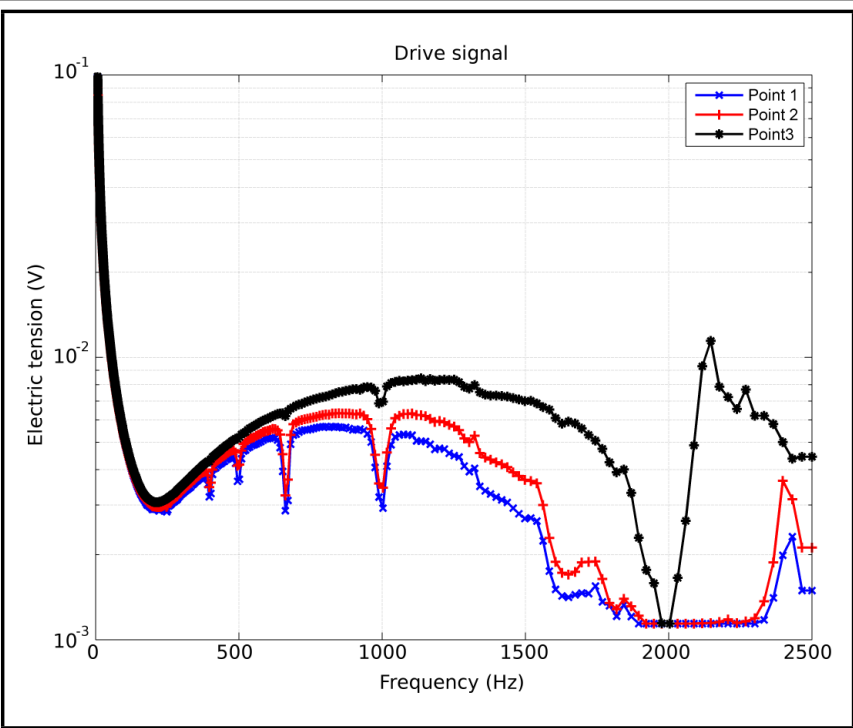

Figure 8. Frequency response of output drive signal for the control points 1, 2 , and 3 .

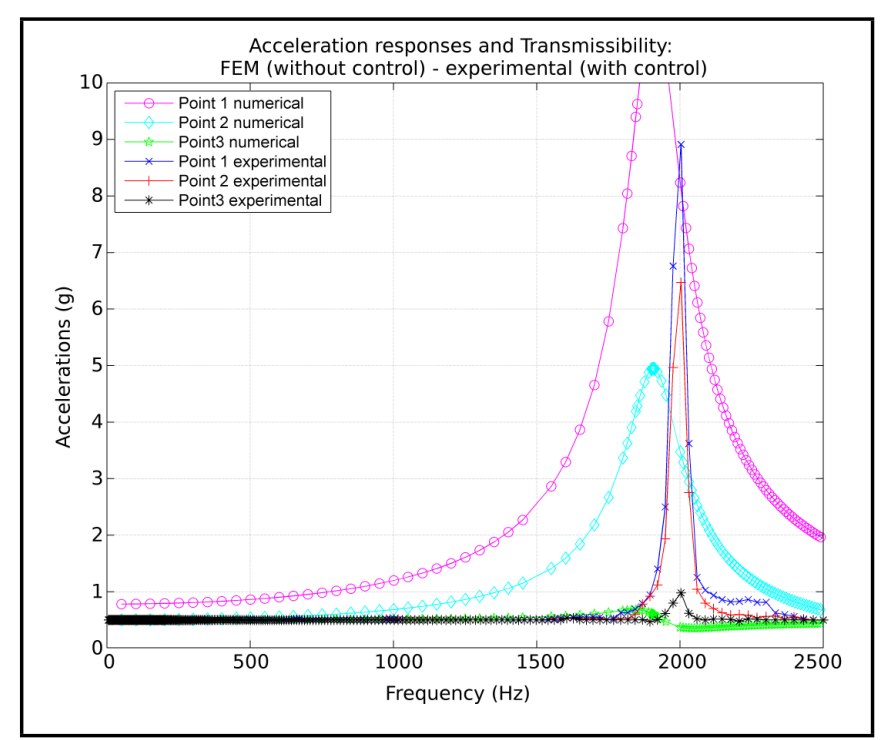

Figure 9. Frequency response/Transmissibility provided by control accelerometer and calculated, for the points 1,2 , and 3 .

reached maximum values of $8.91 \mathrm{~g}, 6.47 \mathrm{~g}$, and $0.97 \mathrm{~g}$, for the control points 1,2 , and 3 , respectively. The vibration level exceeded the value of $0.706 \mathrm{~g}$ in $1869 \mathrm{~Hz}$ (points 1 and 2) and $1976 \mathrm{~Hz}$ (point 3), when the control system aborted the test for safety reasons. By comparing the curves in Fig. 7 and Fig. 8, one can realize that the control system reduced strongly the drive signal four times before the complete signal was aborted, close to the frequency of $2000 \mathrm{~Hz}$. The control system performance explains the different amplitudes for the curves displayed in Fig. 6 and Fig. 7, displayed again together in Fig. 9. The values of transmissibility for the responses calculated using finite elements and for the ones measured with control accelerometer were calculated and are also displayed in Fig. 9 as a function of frequency. A zoomed view of the frequency band $1350-2450 \mathrm{~Hz} \mathrm{~Hz}$ is displayed in Fig. 10. 


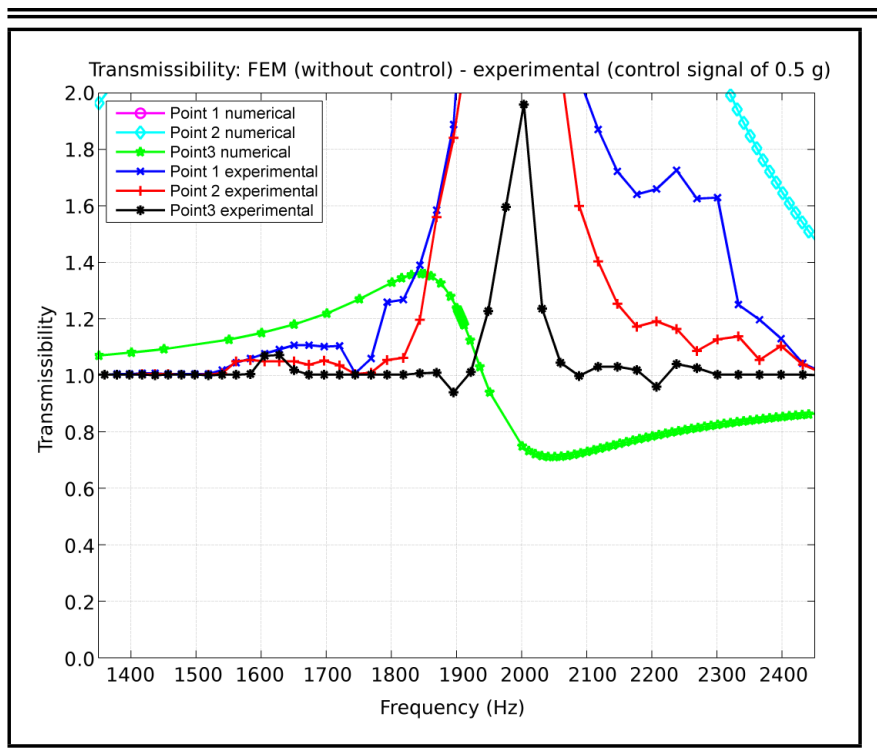

Figure 10. A zoomed view of the frequency band $1350 \mathrm{~Hz}-2450 \mathrm{~Hz}$.

\section{CONCLUSIONS}

Some conclusions about the useful operating frequency range of the fixture can be obtained from the analysis shown in item 4 . The first mode natural frequency in the free-free condition calculated using finite elements, $1738.16 \mathrm{~Hz}$, (see Table 2) indicates a superior limit to the usable frequency range, but the transmissibility curves displayed in Fig. 7 show values close to unity only up to $500 \mathrm{~Hz}$. Only the fixture's regions in the inner circle, which contains the point 3 , will have unitary values of transmissibility up to about $1500 \mathrm{~Hz}$.

However, the acceleration response curves measured in the tests have much smaller amplitudes in the frequency up to $2000 \mathrm{~Hz}$ (although bigger in the peak), due to the control system performance. From Fig. 9, one can conclude that the transmissibility with unitary values is obtained in the three points $(1,2$, and 3$)$ up to $1500 \mathrm{~Hz}$. In this way, the fixture characteristics by itself allow its use up to $1500 \mathrm{~Hz}$ for DUTs fixed in the region limited by the circle that contains point 3 , and up to $500 \mathrm{~Hz}$ for DUTs fixed anywhere on the fixture's top face. This is a very limited operational condition because of the small area of the fixture that can be used. The performance of the control system extends the use up to $1500 \mathrm{~Hz}$ for all regions of the fixture's top face.

Finally, it can be concluded that since the performance of the closed loop control system was able to extend the fixture frequency range, the whole system fixture as well as the control system must be analysed to determine a fixture useful operating frequency range, showing the need of validation tests, like the one conducted in this work. By analysing only the fixture dynamics, one will reach in a much more limited useful operating frequency range. In future works, it is intended to perform same analysis for random and shock input, in addition to the harmonic input investigated here.

\section{REFERENCES}

1 Reddy, T. S. and Reddy, K. V. K. Design and analysis of vibration test bed fixtures for space launch vehicles,
Indian J. Science and Technol., 5 (3), 592-595, (2010). https://dx.doi.org/10.17485/ijst/2010/v3i5/29761

2 Avitable, P. Why you can't ignore those vibration fixture resonances. Sound Vib, 3, 20-26, (1999).

${ }^{3}$ Girard, A., Dupuis, P.E., Lozano, M. Adapter for axial vibrations of large specimens, Proc. European Conference on Spacecraft Structures, Materials \& Mechanical Testing, Noordwijk, The Netherlands, (2005).

${ }^{4}$ Sowjanya, G.P.; Rao, P.D.; Kiran, C.U. Finite Element Analysis of Vibration Fixture made of Aluminum and Magnesium Alloys, IJLTET, 2 (1), 84-89, (2013).

5 Kim, J. Y., Jeong, W. B., Lee, S. B., and Lee, B. H. An experimental approach for structural dynamic modification of fixture in vibration test control, JSME Int. J. Ser. C, 44 (2), 334-340, (2001). https://dx.doi.org/10.1299/jsmec.44.334

${ }^{6}$ Jeong, W. B. and Yoo, W. S. Sensitivity analysis of anti-resonance frequency for vibration test control of a fixture, KSME Int. J., 17 (11), 1732-1738 (2003). https://dx.doi.org/10.1007/BF02983603

7 Mao, Y., Ji, Y., Wang, Q., Huang, H., Yue, X., Yang, Q., and Zhang, C., Technique for dynamic response optimization of shock test fixtures, Proc. International Modal Analysis Conference, Dearborn, USA, (2004).

${ }^{8}$ Qintao, G., Jian, C., Lingmi, Z., Xinqiang, Z., and Yue, Z., Random response analysis based model validation and structural optimization of a vibration fixture, Proc. International Modal Analysis Conference, Orlando, USA (2009).

9 Stephens, C., Fixture Design for Vibration and Shock Test, Technology Training, Inc., California, USA, (2007).

${ }^{10}$ Klee, B. J., Kimball, D. V., and Tustin, W., Vibration and Shock Test Fixture Design: Design, Fabrication, and Evaluation, Tustin Institute of Technology, Santa Barbara, USA, (1971).

11 ISO 5344:2004, Electrodynamic test equipment for generating vibration - methods of describing equipment characteristics, International Organization for Standardization, Switzerland, (2004).

12 Buzdugan, G., Mihailescu, E., and Rades, M., Vibration measuring, Martinus Nijhoff Publishers, Bucharest, Romania, (1986).

13 McConnell, K.G. and Varoto, P.S., Vibration testing: theory and practice, John Wiley \& Sons, New York, USA, (2008).

${ }^{14}$ Cook, R. D., Malkus D. S., and Plesha, M. E., Concepts and applications of finite element analysis, Jonh Wiley \& Sons, New York, USA, (1989). 